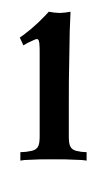

\title{
LA ACREDITACIÓN DE TÍTULOS UNIVERSITARIOS OFICIALES EN EL ÁMBITO DE CIENCIAS DE LA EDUCACIÓN: UNA CUESTIÓN DE ALFABETIZACIÓN ACADÉMICA
}

\author{
(THE ACCREDITATION OF BACHELOR'S AND MASTER'S DEGREES IN \\ EDUCATION: A MATTER OF ACADEMIC LITERACY)
}

Eduardo García-Jiménez

Fernando Guzmán-Simón

Universidad de Sevilla

DOI: $10.5944 / e d u c X X 1.16451$

Cómo referenciar este artículo/How to reference this article:

García-Jiménez, E. y Guzmán-Simón, F. (2016). La acreditación de títulos universitarios oficiales en el ámbito de Ciencias de la Educación: Una cuestión de alfabetización académica. Educación XX1, 19(2), 19-43, doi: 10.5944/educXX1. 16451

García-Jiménez, E. \& Guzmán-Simón, F. (2016). La acreditación de títulos universitarios oficiales en el ámbito de Ciencias de la Educación: Una cuestión de alfabetización académica. [The accreditation of bachelor's and master's degrees in Education: A matter of academic literacy]. Educación XX1, 19(2), 19-43, doi: 10.5944/educXX1.19.2. 16451

\section{RESUMEN}

La publicación de las normas que regulan la ordenación de las enseñanzas oficiales ha llevado a las universidades y agencias de evaluación españolas a un conjunto de retos ligados al diseño, desarrollo y evaluación de los resultados de aprendizaje en el escenario del EEES. En este ámbito, la evaluación de los “Trabajos Fin de Estudios" (TFG o TFM) está aportando información sustancial sobre la diferencia entre los resultados de aprendizaje esperados en un plan de estudios y los realmente logrados por el alumno.

La escritura académica — en forma de TFG y TFM - es una evidencia fundamental para determinar la calidad de los resultados de aprendizaje de un plan de estudios que conducen a un título universitario oficial. No obstante, la evaluación de la escritura académica, como evidencia de la calidad de un título, plantea importantes retos no solo a las universidades que forman a los estudiantes y evalúan sus trabajos de fin de estudios, sino también a las agencias de evaluación que acreditan los títulos. Uno de esos retos es la definición del propio objeto de evaluación — los TFG/TFM - a partir de los diferentes 
prototipos del género discursivo académico "Trabajo Fin de Estudios". Desde una perspectiva socio-discursiva, este artículo propone una descripción de los distintos prototipos de TFG y TFM (intervención educativa, investigación empírica, revisión teórica, investigación histórica) como género en una comunidad discursiva en el área de conocimiento de Educación. Sus características sociales y materiales requieren de los estudiantes el desarrollo de una identidad académica en la escritura que evidencie los resultados de aprendizajes previstos en la legislación.

\section{PALABRAS CLAVE}

Discurso académico; evaluación de la escritura; Trabajo Fin de Grado y de Máster; resultados de aprendizaje.

\section{ABSTRACT}

Publication of the rules governing the organization of official teachings has led to universities and agencies Spanish evaluation to a set of challenges related to the design, development and evaluation of learning outcomes in the setting of the EHEA. In this area, the evaluation of the Bachelor/Master's thesis is providing substantial information on the difference between the expected learning outcomes in curriculum and actually achieved by the student. Academic writing is fundamental to determine the quality of learning outcomes of a curriculum leading to university degree evidence. However, the evaluation of academic writing as evidence of the quality of a title not only poses significant challenges to universities that train students and evaluate their work in order to study but also rating agencies that accredit titles. One of those challenges is the definition of the proper object of evaluation - the Bachelor/Master's Thesis - from different prototypes of academic discourse genre «End of studies work». From a socio-discursive perspective, this article proposes a description of the various prototypes of Bachelor/Master's thesis (educational intervention, empirical research, theoretical review, historical research) gender as a discursive community in the area of knowledge of Education. Social and material characteristics require students to develop academic identity in writing evidencing learning outcomes foreseen in the legislation.

\section{KEY WORDS}

Academic Discourse; Writing Evaluation; Theses; Academic Achievement. 


\section{INTRODUCCIÓN}

La publicación del R.D. 1393/2007, de 29 de octubre, por el que se establece la ordenación de las enseñanzas universitarias oficiales, ha tenido un efecto «bola de nieve» en el sistema universitario español. Por citar algunos de los hitos más importantes de dicho proceso relacionados con este trabajo: quedó sin efecto el catálogo de títulos y, por lo tanto, la definición normativa de la estructura y el contenido de las principales materiales de los diferentes planes de estudios. Como consecuencia de ello, fue necesario que las universidades diseñaran ex novo proyectos de títulos universitarios, que a partir de ese momento formarían parte de un Registro de Universidades, Centros y Títulos (RUCT). También se estableció que aquellas enseñanzas que cumplieran determinados requisitos y criterios de calidad conducirían a títulos de grado y máster con un carácter oficial y, por ende, aquellas que no los cumplieran se convertirían en títulos propios de las universidades. De igual modo, los proyectos de títulos fueron sometidos a una acreditación previa (verificación) a su implantación y, tras ésta, a una nueva acreditación (reacreditación) que determinaba la permanencia o no del título en el RUCT.

La nueva ordenación de las enseñanzas adoptaba como principio la profundización «en la concepción y expresión de la autonomía universitaria» y dictaba una serie de medidas que «flexibilizan la organización de las enseñanzas universitarias, promoviendo la diversificación curricular y permitiendo que las universidades aprovechen su capacidad de innovación, sus fortalezas y oportunidades» (R.D. 1393/2007. Preámbulo). No obstante, en el Anexo I de dicha norma, así como en del R.D. 810/2010 que lo modificaba, se recogía la "Memoria para la solicitud de verificación de títulos oficiales" que configuraba el proyecto de título oficial que debían presentar las universidades. Entre los elementos que habían de aportar las universidades estaba la estructura de las materias que constituían la propuesta de un título de grado o de máster. En ella, se incorporaba un tipo de materia que -salvo en el área de conocimientos de Ingeniería y Arquitectura- representaba una verdadera novedad para la mayoría de los títulos preexistentes en las restantes áreas y ámbitos de conocimiento (entre ellos, los del ámbito de la Educación): los Trabajos Fin de Grado y los Trabajos Fin de Máster. En consecuencia, los centros y departamentos universitarios se enfrentaban al diseño, implantación y evaluación de un tipo de materia cuyos rasgos característicos eran desconocidos para la mayoría de sus equipos de gobierno y de su personal académico.

La publicación del R.D. 1393/2007 y sus modificaciones posteriores tuvo también efectos en otros agentes de interés en el sistema universitario. En este sentido, nos referimos a los organismos externos a las universidades que debían primero verificar los proyectos de título y, después, acreditar 
su implantación. Así, la Agencia Nacional de Evaluación y Acreditación (ANECA) y los órganos de evaluación que las comunidades autónomas habían creado, tenían el mandato de establecer los «protocolos de evaluación necesarios para la verificación y acreditación de acuerdo con los mencionados criterios y directrices» (art. 24), y conforme a lo dispuesto en dicho real decreto. Al igual que las universidades, los órganos de evaluación de ámbito nacional y autonómico (en adelante agencias de evaluación) también debían afrontar encomiendas para las que no había registros históricos. Entre estas estaba la de verificar y acreditar enseñanzas a partir de los resultados de aprendizaje esperados y logrados por los estudiantes en el nuevo escenario del Espacio Europeo de Educación Superior (EEES), y un nuevo sistema de medida basado en los créditos europeos (ECTS).

La adopción de este sistema [ECTS] constituye una reformulación conceptual de la organización del currículo de la educación superior mediante su adaptación a los nuevos modelos de formación centrados en el trabajo del estudiante. Esta medida del haber académico comporta un nuevo modelo educativo que ha de orientar las programaciones y las metodologías docentes centrándolas en el aprendizaje de los estudiantes, no exclusivamente en las horas lectivas (BOE, 2013, p. 34355).

En síntesis, universidades y agencias de evaluación, respectivamente, tenían que diseñar y verificar enseñanzas centradas en el trabajo de los estudiantes, así como evaluar y acreditar el producto de ese trabajo. El referente de evaluación y acreditación, a utilizar por ambos tipos de instituciones para los diferentes niveles de enseñanzas, se concretó primero en el Anexo I del R.D. 1393/2007 y, después, en forma de Marco Español de Cualificaciones para la Educación Superior (MECES), recogido en el R.D. 1027/2011, de 15 de julio. El MECES venía a desarrollar un conjunto de descriptores o «colección de resultados del aprendizaje que caracteriza un determinado nivel en un marco de cualificaciones» (art. 2). De este modo, los resultados de aprendizaje («aquello que se espera que un estudiante conozca, comprenda o sea capaz de hacer» [art. 2]) se convirtieron en la base de una cualificación profesional. De este modo, «cualquier título, diploma o certificado emitido por una institución educativa» debía acreditar que un estudiante había adquirido un conjunto de resultados del aprendizaje, tras «haber superado satisfactoriamente un programa de formación en una institución legalmente reconocida en el ámbito de la educación superior» (art. 2).

En un título como el de Grado de Maestro en Educación Primaria, las universidades tenían que evaluar la formación del estudiante a partir de sus resultados de aprendizaje, antes de emitir dicho título. Para ello, debían 
adoptar como referente los descriptores correspondientes a los títulos de nivel de Grado establecidos en el MECES. De forma complementaria, las agencias de evaluación tenían que comprobar en qué medida las enseñanzas conducentes a ese título habían desarrollado los resultados de aprendizaje en los estudiantes, que cabría esperar para un Grado de Maestro en Educación Primaria.

En definitiva, las universidades y las agencias se enfrentan a un conjunto de cuestiones ligadas al diseño, desarrollo y evaluación de los resultados de aprendizaje. De ellas, en el presente trabajo, vamos a ocuparnos de aquellas que se centran en la evaluación de los resultados de aprendizaje.

\section{LA EVALUACIÓN DE LOS RESULTADOS DE APRENDIZAJE}

La evaluación del aprendizaje de los estudiantes en una materia o asignatura es consustancial a la vida universitaria, de modo que se ha convertido en una rutina más en el desarrollo de un plan de estudios. Con la excepción de los trabajos fin de estudios y de la tesis doctoral (sin entrar en detalles sobre sus limitaciones como evidencias), en el sistema universitario español no ha existido una praxis sistemática de evaluación de los resultados del aprendizaje logrados por un estudiante al término de su período de formación. Se había partido del supuesto de que un estudiante que completaba satisfactoriamente todas las asignaturas de un plan de estudios había adquirido los resultados de aprendizaje previstos. No obstante, no se había establecido un procedimiento para comprobarlo. De igual modo, dado que hasta 2007 el Gobierno de España garantizaba la «calidad intrínseca» de los planes de estudios publicados en el Boletín Oficial del Estado, «no tenía sentido» cuestionar la calidad de los estudios impartidos en las universidades a partir de la evaluación de los resultados de aprendizaje logrados por los egresados.

El efecto «bola de nieve», provocado por la normativa que ha regulado la incorporación del sistema universitario español al EEES, ha cambiado dicho escenario. En el ámbito de los resultados de aprendizaje de los alumnos considerados a título individual, la evaluación de los trabajos fin de estudios (de Grado [TFG] o de Máster [TFM]) está aportando información sustancial sobre la diferencia entre los resultados de aprendizaje esperados en un plan de estudios y los realmente logrados por el alumno. De igual modo, otras iniciativas desarrolladas por las universidades para la evaluación de las denominadas competencias transversales también están ayudando a medir la distancia entre lo logrado y lo esperado en ese tipo de competencias. A modo de ejemplo, cabe mencionar aquí las diferentes iniciativas recogidas en el número monográfico de la Revista de Docencia 
Universitaria (REDU) publicado en 2010 y titulado "La evaluación de competencias". (AA.VV., 2010).

En el ámbito de la evaluación de la calidad de los resultados de aprendizaje alcanzados por los egresados de un título, el desarrollo por parte de las agencias de evaluación de protocolos para la acreditación de los títulos está poniendo en valor el papel de dichos resultados. Así, el Consorcio Europeo de Acreditación (ECA, en sus siglas en inglés), en los Principles regarding outcomes in accreditation procedures, señala que sus miembros (entre los que figuran la mayoría de las agencias de evaluación españolas) "están de acuerdo en que los resultados de aprendizaje pueden ser tomados en consideración durante cualquier proceso de evaluación en educación superior y que este hecho realza el enfoque centrado en el estudiante". En ese mismo documento, los miembros de ECA acuerdan que "tener en cuenta los resultados de aprendizaje en los procesos de acreditación contribuye al reconocimiento mutuo de las decisiones de acreditación [que afectan a títulos de diferentes países europeos]” (ECA, 2012).

Con objeto de orientar la utilización de los resultados de aprendizaje en los procesos de acreditación de títulos, ECA ha establecido siete principios que vienen a destacar que «los organismos de acreditación deben considerar los resultados de aprendizaje en sus evaluaciones» (principio 1), «evaluar si los resultados de aprendizaje están alineados con el Marco Nacional de Cualificaciones [MECES, en España]» (principio 2), o «evaluar si el diseño y el contenido del plan de estudios permiten a los estudiantes lograr los resultados del aprendizaje previstos y si las instituciones de educación superior utilizan procedimientos adecuados para evaluar dichos resultados del aprendizaje previstos» (principio 5) (ANECA, s.f., pp. 58-59).

Para articular los procesos de acreditación en el sistema universitario español, las agencias de evaluación se han apoyado en un documento titulado "Criterios y directrices de evaluación para la acreditación de títulos oficiales de Grado, Máster y Doctorado», elaborado por la Red de Agencias de la Calidad de las Universidades (REACU). En dicho documento, la evaluación de los resultados de aprendizaje se recoge en el "Criterio 6. Resultados de aprendizaje», que establece: «Los resultados de aprendizaje alcanzados por los titulados son coherentes con el perfil de egreso y se corresponden con el nivel del MECES (Marco Español de Cualificaciones para la Educación Superior) de la titulación» (REACU, 2014, p. 9). Además, en el documento de REACU se indica de manera explícita (p. 10): 
En ningún caso puede conseguirse la acreditación si se obtiene la calificación de "no se alcanza" en alguno de los siguientes criterios:

a) Criterio 4. Personal académico.

b) Criterio 5. Recursos y apoyo al estudiante.

c) Criterio 6. Resultados de aprendizaje.

En la concreción de sus protocolos de evaluación para la acreditación de títulos oficiales de Máster y de Grado, las agencias de evaluación identifican tanto los aspectos concretos a valorar como las evidencias en las que apoyar sus decisiones de acreditación. Así, por ejemplo, la Fundación Madrid recoge entre los aspectos que valora la «adecuación de los TFG/TFM a las características del título» $(2014$, p. 38) y, al hacer referencia a la información en la que se basa la evaluación, se indica que el panel de evaluación externa seleccionará dichos trabajos. La AQU-Catalunya precisa que es necesario recoger muestras de las ejecuciones de los estudiantes para valorar sus resultados de aprendizaje y, para ello, las universidades deberán «preparar una selección de evidencias de las pruebas evaluativas de los estudiantes en el marco del TFG/TFM (...). La selección de las pruebas escritas, trabajos y/o informes debe realizarse de modo que cubra el espectro de calificaciones» (2013, p. 46).

Las agencias de evaluación, según cabe deducir de los documentos que elaboran para sus paneles de evaluación externa (y para las universidades y la sociedad en general), vienen a establecer que la escritura académica -en forma de TFG y TFM- es una evidencia fundamental para determinar la calidad de los resultados de aprendizaje de un plan de estudios que conducen a un título universitario oficial. No obstante, la evaluación de la escritura académica como evidencia de la calidad de un título plantea importantes retos no sólo a las agencias de evaluación, sino también a las propias universidades que forman a los estudiantes y evalúan sus trabajos fin de estudios.

La evaluación de los resultados de aprendizaje a través de las producciones escritas realizadas por los alumnos en forma de TFG/TFM plantea diferentes retos. Estos están relacionados con la definición del objeto de evaluación desde los diferentes géneros académicos, la elaboración de criterios de evaluación a partir de los resultados de aprendizaje esperados, la búsqueda de referentes de lo que es un "Trabajo Fin de Estudios" bien hecho o la resolución de los conflictos de interés asociados a la evaluación y calificación de los trabajos. En el presente artículo vamos a ocuparnos de la definición del objeto de evaluación a partir de los diferentes prototipos del género dis- 
cursivo académico "Trabajo Fin de Estudios", dado que de su resolución depende en buena medida el modo en que se enfoquen los demás retos.

\section{DEFINICIÓN DEL OBJETO EVALUACIÓN}

Un primer reto al que se enfrenta la evaluación está relacionado con la identificación del objeto de evaluación, es decir, aquel aspecto sobre el que se centra la valoración del aprendizaje de los estudiantes (Rodríguez Gómez e Ibarra Sáez, 2011). En la revisión realizada en este artículo, el objeto de evaluación son los resultados del aprendizaje logrados por los estudiantes. De una forma más concreta, y considerando el medio de evaluación, aquellos resultados que se evalúan a partir del texto escrito presentado por los estudiantes en forma de "Trabajo Fin de Estudios".

\section{NATURALEZA DE LA ESCRITURA ACADÉMICA Y ENFOQUES}

En las últimas décadas, la escritura académica ha sido analizada desde la perspectiva tanto de las investigaciones sobre las Academic Literacies (ACLITS) en el Reino Unido como la Writing across to curriculum (WAC) y Writing in the disciplines (WID) en USA (cfr. Russell, Lea, Parker, Street y Donahue, 2009). Ambas perspectivas han delimitado un campo de investigación sobre los tipos de discurso y sus usos en el entorno académico. Desde el marco desarrollado por ACLITS (Russell, Lea, Parker, Street, y Donahue, 2009), nuestro enfoque ha planteado tanto la descripción de un tipo de discurso (trabajo fin de estudios) como su adquisición y uso en un entorno académico español.

El concepto escritura académica debe enmarcarse en el concepto más amplio de alfabetizaciones académicas, desarrollado por los Nuevos Estudios de Literacidad (Barton, 1994; Street, 1984). No obstante, la configuración de dicho concepto fue elaborada por Lea y Street (1998; 2006), donde la escritura académica de los estudiantes era el resultado de una práctica social desarrollada en la Universidad (Gee, 1996; Barton, Hamilton, y Ivani冈, 2000; Lillis, 2001; Wingate y Tribble, 2012).

El reto del proceso de enculturización (Prior y Bilbro, 2011) académica en la actualidad es el desarrollo de las alfabetizaciones académicas necesarias para que un estudiante pueda ser parte de una comunidad de práctica. Este hecho hace que muestre tanto habilidades de estudio (la capacidad de transferir sus conocimientos sobre la lectura y la escritura de un contexto a otro) y cierto grado de socialización académica (aprendizaje a comunicarse oralmente o por escrito en diversos géneros discursivos prototípicos 
de una disciplina) como la alfabetización académica (la suma de todos estos elementos que permite al estudiantes escribir un ensayo en un contexto disciplinar concreto y construir su propio discurso crítico). La alfabetización académica en un nuevo contexto social (Street, 2004) como el actual de la universidad europea puede ser definido como un

(...) proceso de enseñanza que puede (o no) ponerse en marcha para favorecer el acceso de los estudiantes a las diferentes culturas escritas de las disciplinas. Es el intento denodado por incluirlos en sus prácticas letradas, las acciones que han de realizar los profesores, con apoyo institucional, para que los universitarios aprendan a exponer, argumentar, resumir, buscar información, jerarquizarla, ponerla en relación, valorar razonamientos, debatir, etcétera, según los modos típicos de hacerlo en cada materia. Conlleva dos objetivos que, si bien relacionados, conviene distinguir: enseñar a participar en los géneros propios de un campo del saber y enseñar las prácticas de estudio adecuadas para aprender en él (Carlino, 2013, p. 370).

\section{CARACTERÍSTICAS DE LAS COMUNIDADES DISCURSIVAS ACADÉMICAS}

La noción «comunidad» hace referencia a un contexto alfabetizador donde una acción (leer y escribir) es producida en un marco (universidad), y donde el agente (estudiante) persigue una finalidad comunicativa particular. Barton (1994) introduce el concepto de comunidad discursiva como «un grupo de personas que poseen textos y prácticas en común» (p. 57). Dichas prácticas parten de actividades cotidianas en nuestra sociedad, cuya forma y estructura caracteriza una determinada práctica social (Barton, 1991), así en el ámbito de la alfabetización académica los distintos géneros son reconocidos, interpretados y producidos por los miembros que componen dicha comunidad (Swales, 1990; 2004).

La comunidad discursiva académica, como práctica social de escritura, comparte en su heterogeneidad ciertas convenciones sobre qué (tópico), a quién (tenor) y a través de qué medio comunicar (medio). Dichas convenciones se convierten en uno o varios géneros discursivos a partir de las prácticas sociales e históricas en un colectivo como, por ejemplo, el artículo de investigación (Hyland, 2009, pp. 67-78; Swales, 1990, pp. 110-176) o la tesis doctoral (Swales, 2004, pp. 99-144), entre otros.

Partiendo de los presupuestos anteriores, este artículo asume un modelo socio-discursivo, donde las condiciones sociales de producción e interpretación son fundamentales para entender los elementos que han hecho 
posible la producción y la comprensión de un texto. A partir del modelo desarrollado por Fairclough (1989) consideramos, de una parte, que cualquier género discursivo o texto, como práctica discursiva social, depende del contexto cultural donde una institución explicita las condiciones de producción e interpretación de la comunicación escrita (por ejemplo, la Universidad). Por otro lado, el contexto de situación determina claramente cuáles son los objetivos concretos de la comunicación escrita, a quién va dirigida y a través de qué medio (por ejemplo, al final de un grado los alumnos escriben un trabajo final de estudios).

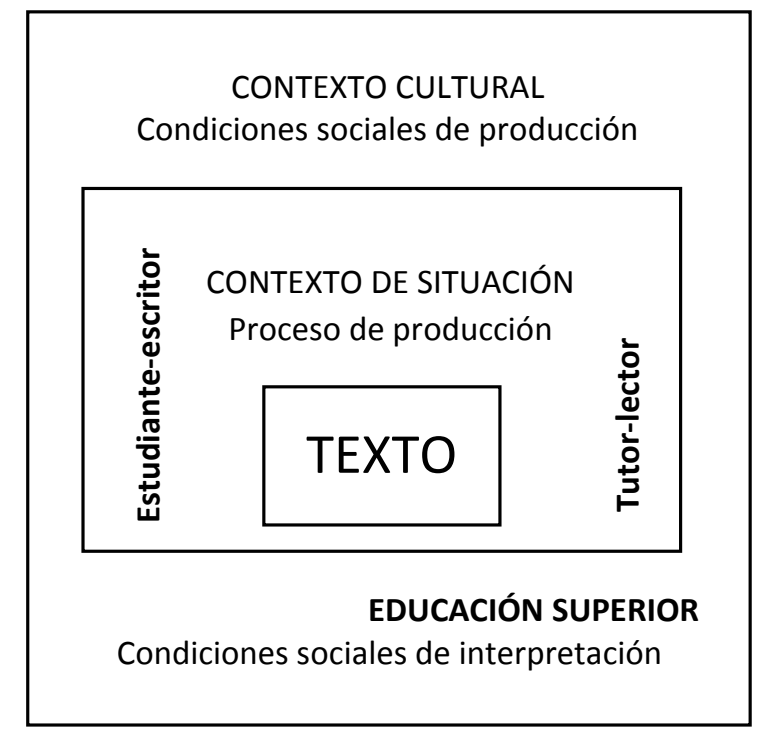

Figura 1. Modelo socio-discursivo de Fairclough (1989)

Fuente: Fairclough (1989, p. 25) y Lillis (2001, p. 35).

En un contexto universitario actual, los estudiantes de Grado y Máster están inmersos en múltiples alfabetizaciones y diversos sistemas de escritura (Prior, 1998; Russell y Yañez, 2003) en ámbitos muy distintos (hogarfamiliar, trabajo, universidad, entorno, etc.) (Ivani囚 et al., 2009). La tensión creada entre las alfabetizaciones de varios ámbitos en las que participa el estudiante universitario no facilita una correcta enculturación académica, donde la escritura se convierte en la evidencia más visible de este proceso. En particular, la generalización de la escritura de trabajos fin de estudios (TFG y TFM) ha mostrado diversas carencias que presentan los alumnos en su formación universitaria. En consecuencia, aquellos alumnos que no desarrollan en un grado aceptable las convenciones de la escritura académica pueden ser excluidos de su comunidad de práctica de referencia. 


\section{DEFINICIÓN DE UN GÉNERO DISCURSIVO ACADÉMICO}

En el ámbito de la Educación, la escritura académica asume un carácter multimodal, en el que se mezclan en un mismo plano del texto elementos verbales, numéricos y visuales (Kress, 2003). La construcción de un discurso discontinuo en este tipo de trabajo académico requiere también de un claro desarrollo de la habilidad escritora que posibilita este tipo de producción. De ahí que Swales (1990) subraye que un género discursivo es un evento con un propósito comunicativo que se concreta en diversos prototipos. Cada uno de ellos recoge un contenido, una estructura y una serie de elementos formales presentes en el texto y son configurados en el seno de una comunidad discursiva particular (pp. 45-57).

Por otro lado, English (2011) incorpora a esta definición una dimensión pedagógica consiguiendo que la escritura académica se convierta en parte del aprendizaje de una materia o asignatura. Este planteamiento es, quizás, el más adecuado para abordar el TFG y el TFM como géneros discursivos (con sus diversos prototipos) en un contexto de aprendizaje académico. En este sentido, los trabajos fin de estudios son un producto semiótico donde interaccionan los participantes en un evento comunicativo (Kress y Van Leeuwen, 1996).

En este sentido, los distintos rasgos que definen un género discursivo han sido clasificados por Fiona English (2011). Su propuesta parte de la elección de un género discursivo (o prototipo) que está determinado por el medio de difusión, su temática, a quién va dirigido, la estructura y organización de los materiales textuales (English, 2011). Esta circunstancia caracteriza el diseño, la producción y la distribución del propio discurso. A este conjunto de elementos que definen un prototipo de género discursivo es denominado por English (2011) orientación contextual.

Del mismo modo, un segundo conjunto de rasgos es denominado orientación discursiva. La escritura de diversos prototipos de discursos en un contexto académico permite al alumno desarrollar su identidad como escritor, siendo marcadas las orientaciones institucionales o académicas que debe seguir para formar parte de una comunidad de práctica. El tercer elemento señalado por English (2011) es la orientación temática del discurso escrito. Este elemento caracteriza al género discursivo en la medida en que relaciona la temática del texto y su organización textual. Por último, la orientación semiótica de un texto determina en un género discursivo el material textual, el modo y el medio en que se difunde (English, 2011). 


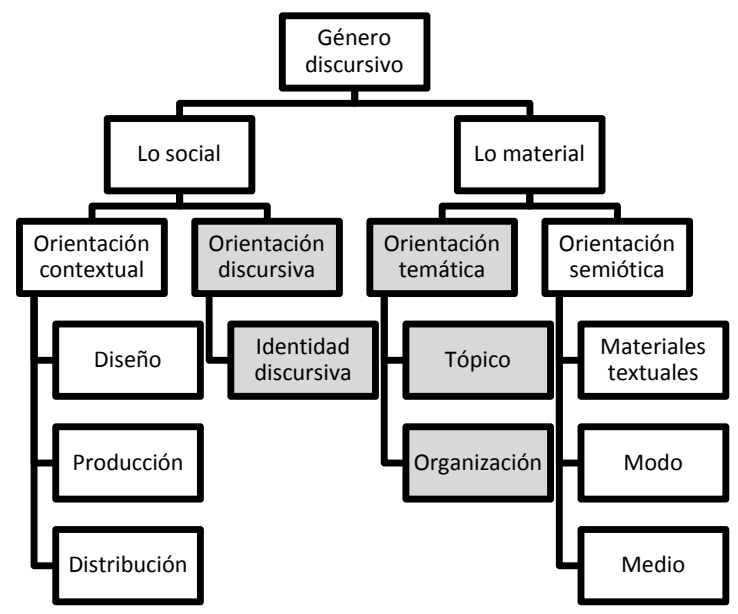

Figura 2. Clasificación de los rasgos característicos de los géneros discursivos y sus prototipos

Fuente: Adaptación de English (2011, p. 88).

\section{EL “TRABAJO FIN DE ESTUDIOS” COMO GÉNERO DISCURSIVO}

Los alumnos deben afrontar la escritura de los trabajos de fin de estudios incorporando no sólo los conocimientos adquiridos en sus años de Grado y Máster. Por el contrario, dicho trabajo ha de reflejar en su escritura las expectativas de una investigación académica, los propios procesos de elaboración de dicha escritura y las convenciones formales y de estilo características de la escritura académica en un trabajo fin de estudios. Del mismo modo, dichas características han de estar circunscritas a un área de conocimiento y disciplina concreta (Tardy, 2009). En particular, los TFG y TFM en Educación poseen una amplia diversidad o prototipos debido a su propia naturaleza interdisciplinaR.D.e hecho, podemos anotar hasta cinco subtipos o subgéneros de TFG y TFM en este ámbito de las Ciencias Sociales. Por otro lado, delimitar las diferencias que hallamos entre un TFG y un TFM, y de estos con una tesis doctoral, resulta de vital importancia para un correcto desarrollo de la escritura académica de los distintos alumnos.

Con el fin de incorporar a un estudiante universitario a una comunidad discursiva académica de una disciplina concreta, este debe desarrollar el conocimiento del género (Tardy, 2009) que persigue tanto elementos sociales como materiales (English, 2011). En particular, el primero de ellos está compuesto por la orientación contextual y la discursiva y, en ambos casos, son dimensiones que están predefinidas con antelación por el medio académico. Así, la orientación contextual hace referencia al diseño, produc- 
ción y distribución del TFG y el TFM, atendiendo a las características de cuándo se realiza ( $4 .^{\circ}$ curso de Grado y final de Máster), con qué diseño formal (características descritas en las distintas guías universitarias) y cómo se distribuye (según la universidad, a través de los repositorios de TFG y TFM). Del mismo modo, la orientación discursiva queda delimitada en este contexto por el propósito del autor (obtener el título de Grado), el proceso llevado a cabo en el último año/etapa de estudios, la identidad y rol escritor que debe desarrollar como autor y, por último, la agencia (universidad) quien solicita dicho trabajo escrito. En definitiva, la dimensión social de la escritura del TFG y el TFM viene determinada por la normativa universitaria actual.

Por otro lado, la dimensión material del género discursivo TFG y TFM posee otros dos aspectos: la orientación temática y la semiótica. Ambas dimensiones representan buena parte de la elección del autor del TFG y TFM como de su tutor-profesor. En este caso, el tópico o temática determinará la estructura del prototipo de género discursivo utilizado. También, estas características delimitarán qué modo y qué materiales textuales son utilizados para facilitar la comunicación (gráficos, tablas, fotografías, grabaciones, etc.).

En la tabla 1 se han anotado, sin ánimo de ser exhaustivos, diversos prototipos del género discursivo académico «Trabajo fin de estudios». Los prototipos aquí presentados pueden completarse o reformularse en otros términos; así, por ejemplo, hay universidades que recogen un prototipo específico para el Diseño de materiales educativos, en otras lo hacen para la Evaluación de programas en educación y en otras para la Innovación educativa (diferenciada de la intervención). En cualquier caso, los prototipos presentados en la tabla 1 (intervención educativa, investigación empírica, revisión teórica, investigación histórica) son una aproximación a los rasgos temáticos y sociales que vienen determinados por el tópico educativo en la escritura académica. Dicho cuadro recoge, a modo de ejemplo, lo que entendemos ha de concretarse en cada manual de elaboración del TFG y/o del TFM en las Facultades de Ciencias de la Educación y en los Centros de Escritura Académica de las distintas universidades.

En las descripciones recogidas en la tabla 1 no se han incorporado algunos componentes de los "Trabajos Fin de Estudios". De este modo, la Introducción, la Discusión o las Conclusiones presentan escasas diferencias entre sí. En el primer caso, en la "Introducción" justifica la necesidad o la demanda que requiere el diseño/desarrollo de un proyecto de intervención educativa, la investigación empírica, la revisión teórica o la investigación histórica que va a llevarse a cabo. En el segundo caso, la "Discusión" analiza críticamente y según corresponda a cada prototipo los hallazgos obtenidos 
con la intervención educativa, los resultados de la investigación empírica, los principios, modelos, resultados de meta-análisis y otras formulaciones teóricas realizadas o los argumentos que explican o predicen los hechos del pasado. En el tercer caso, las "Conclusiones" incorporan la revisión crítica de los objetivos propuestos y las hipótesis de investigación en el "Trabajo Fin de Estudios". De igual modo, el último apartado recoge las limitaciones y las líneas de investigación futuras de los distintos prototipos de los TFG Y TFM. 
LA ACREDITACIÓN DE TÍTULOS UNIVERSITARIOS OFICIALES EN EL ÁMBITO DE CIENCIAS..

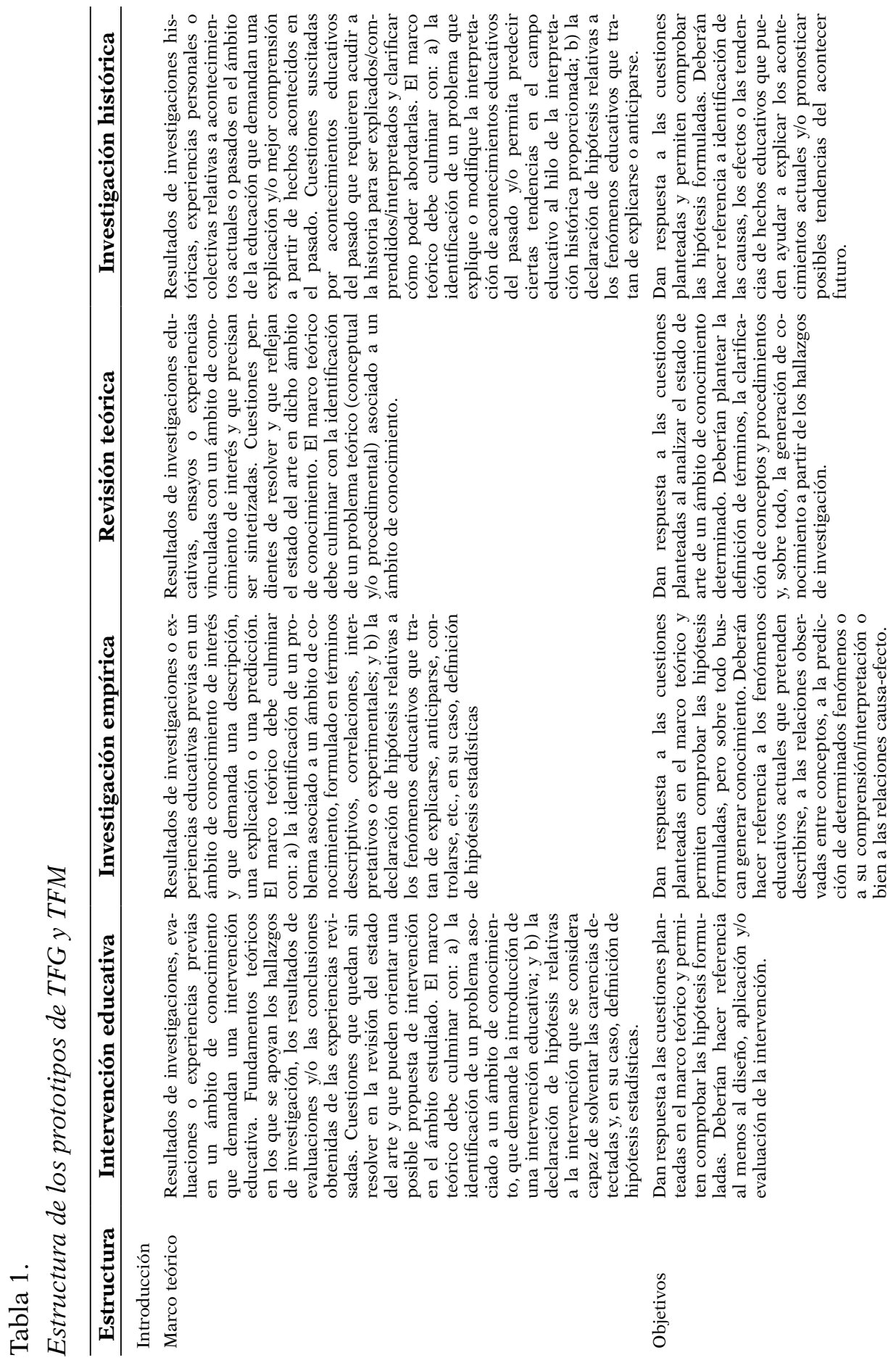




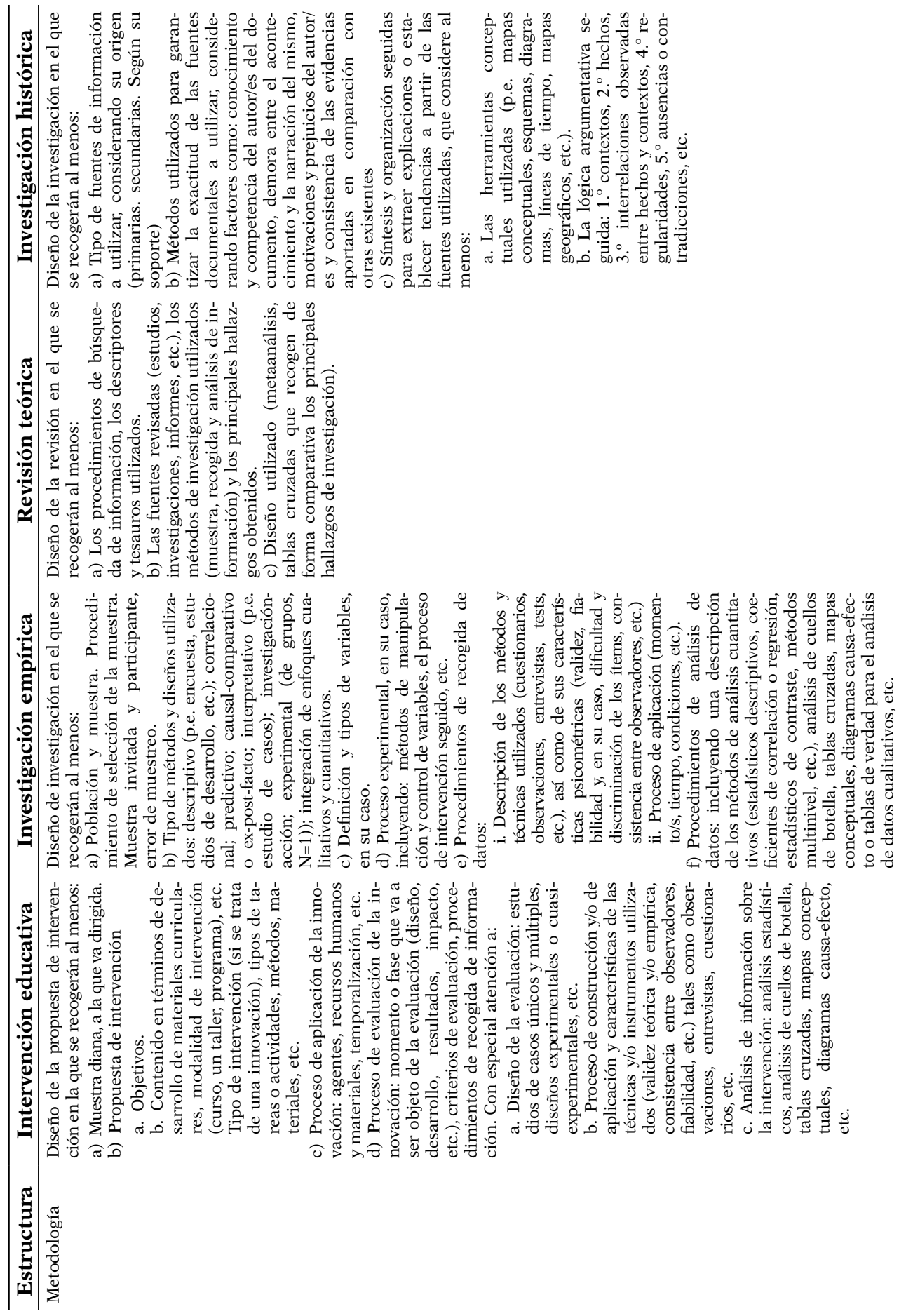




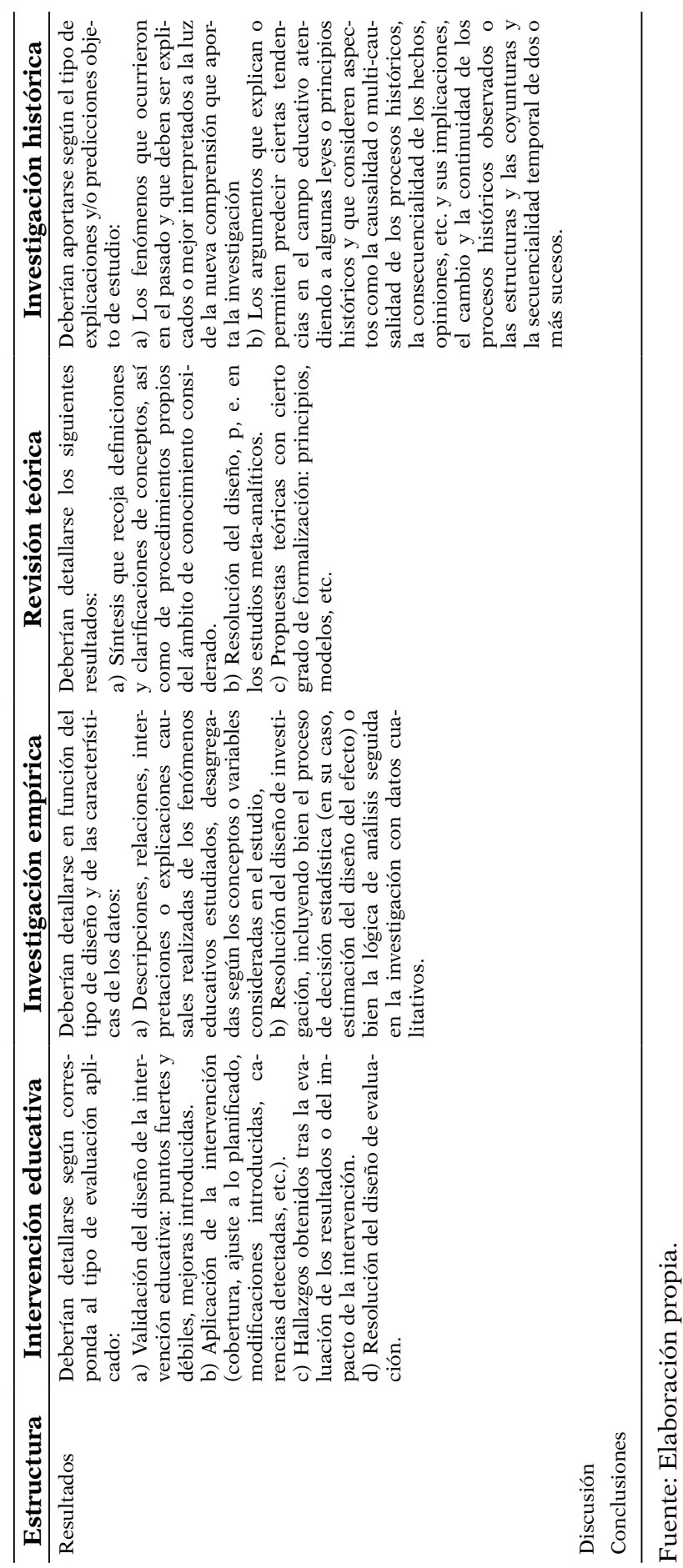




\section{EL PROCESO DE APRENDIZAJE DEL TFG Y TFM EN LA UNIVERSIDAD}

A diferencia de las investigaciones de WAC de Swales (2004), Devitt (2004) y Carter, Ferzli y Weibe (2004), la labor pedagógica de los géneros discursivos académicos desde el enfoque ACLITS ha profundizado sobre las prácticas de evaluación y sus efectos en el aprendizaje (Lillis et al., 2015). Este hecho subraya la necesidad no sólo de cambiar el foco del análisis sobre el aprendizaje del género discursivo, sino la transformación de las prácticas metodológicas con las que se enseña. Así, una auténtica alfabetización académica atendería a una amplia variedad de tipos de textos que se producen en la Universidad, no sólo a aquellos que han dominado desde el punto de vista de la evaluación (Russell et al., 2009). El análisis del British Academic Written English (BAWE) realizado por Nesi y Gardner (2012; 2013) ha permitido la categorización de los distintos géneros discursivos académicos en trece familias distribuidas en cinco funciones. Para el contexto español, dichas funciones han sido adaptadas por Corcelles et al. (2015) y concretado en los siguientes apartados:

a) Conocimiento disciplinar (apuntes, explicación, ejercicios);

b) Análisis crítico (crítica, ensayo, debate);

c) Reflexión personal (apropiación personal);

d) Investigación (revisión de la literatura, informe metodológico e informe de investigación);

e) Práctica profesional (estudio de caso y géneros profesionales) (pp. 554-557).

Según los datos del reciente estudio de Corcelles et al. (2015) y Álvarez y Yániz (2015), la escritura académica menos frecuente, en opinión de profesores y alumnos, es la escritura con las funciones de argumentación crítica y de investigación. Por el contrario, las tareas de escritura de los estudiantes en la universidad generalmente son los exámenes, los resúmenes y la toma de apuntes (Guzmán-Simón y García-Jiménez, 2014). La perspectiva del aprendizaje de los géneros discursivos de ACLITS conlleva un proceso continuo donde la institución académica demanda unos resultados de aprendizaje que posibiliten al alumnado formar parte de una comunidad discursiva académica. De ahí, la necesidad de potenciar en las aulas escrituras que desarrollen las funciones del análisis crítico, la investigación y la reflexión personal. En consecuencia, la escritura académica no reside en la clase de uno $\mathrm{u}$ otro profesor o asignatura, sino que trasciende a estos y pertenece a un nuevo requerimiento institucional. 


\section{DISCUSIÓN/CONCLUSIONES}

Las páginas de este artículo presentan algunas prácticas escasamente desarrolladas en el sistema universitario español. En nuestro estudio hemos constado cómo no existe una tradición basada en la evaluación de los resultados de aprendizaje en un contexto de la acreditación de las enseñanzas universitarias. Del mismo modo, tampoco hay una literatura que aborde la evaluación de la escritura académica como parte de dicho proceso, por lo que este artículo ha tratado de establecer una conexión entre tres conceptos fundamentales: la acreditación de enseñanzas conducentes a títulos oficiales de Grado y Máster, la evaluación de resultados de aprendizaje, y la evaluación de la escritura académica a través de los trabajos fin de estudios.

Siguiendo la tradición de ABET (http://www.abet.org/accreditation/), fundada en 1932 entre otros fines para la acreditación en el ámbito de las ingenierías, las agencias de evaluación están utilizando las producciones de los alumnos (entre ellas, los trabajos fin de estudios) como evidencia para evaluar los resultados de aprendizaje. Estas producciones escritas representan una muestra significativa del grado en que los estudiantes comprenden determinados conceptos y procedimientos, y los aplican para resolver problemas, extraer inferencias y comunicar los resultados obtenidos. En consecuencia, cuando se evalúa un TFG o TFM, se accede al nivel de alfabetización académica del alumno.

Una evaluación de la escritura académica que aportase la información necesaria para valorar el nivel de alfabetización académica del alumno requeriría determinar tanto el ajuste entre la producción escrita del alumno como los requisitos establecidos. Esto necesitaría la

a) Definición de los prototipos de géneros discursivos objeto de evaluación;

b) Identificación de los criterios y otros elementos del proceso de evaluación (referentes, instrumentos, etc.);

c) Aplicación de los requisitos establecidos y la emisión de una calificación.

No obstante, con el establecimiento de estos requisitos no se garantizaría la alfabetización académica de los alumnos universitarios. Para ello, es necesario construir en primer lugar una conciencia de género discursivo en el seno de la comunidad discursiva académica. Esto es especialmente importante en el caso de los trabajos fin de estudios, dado que en España no ha existido una tradición previa y, en el ámbito europeo, no existe un acuerdo 
en la definición de los prototipos asociados a géneros discursivos asociados a los trabajos fin de estudios (Kruse, 2012; 2013). Los estudios españoles (Corcelles, Oliva, Castelló, y Milian, 2015; Álvarez y Yániz, 2015) evidencian cómo en las universidades estudiadas son escasas las tareas de escritura que transformen el conocimiento y conlleven un proceso de investigación (Guzmán-Simón y García-Jiménez, 2015).

En segundo lugar, la reciente implantación de los TFG y TFM requiere una amplia difusión entre el alumnado; es decir, en qué consiste un TFG/TFM y cuáles son los requisitos para su evaluación. En este sentido, se hace necesario formar a los profesores-tutores y a los estudiantes-escritores en los diferentes prototipos, invitándoles a participar igualmente en el proceso de evaluación.

La identidad académica del alumnado en una disciplina se construye a partir de la escritura, dado que la escritura es una estrategia de aprendizaje (Ivanič, 1998). En líneas generales, la escritura académica en las aulas españolas tiene un carácter periférico. Así, la labor docente es aplicada en los extremos (Carlino, Iglesia, y Laxalt, 2013) donde la retroalimentación no es elaborada y tampoco está ligada a la propia calificación de la tarea.

En otros contextos universitarios que se han enfrentado a este problema (Ganobcsic-Williams, 2006; Bazerman et al., 2005) la creación de centros de escritura académica (Writing Center) ha sido un instrumento valioso. Los Centros de Escritura ofrecen recursos y asesoramiento sobre la escritura académica en las diversas disciplinas. Su finalidad es el asesoramiento a profesores y alumnos (cursos, tutorías, publicaciones, etc.) sobre la escritura académica a partir de creación de guías de escritura, documentos de lectura y cursos de formación que faciliten el manejo de diferentes recursos disponibles. Algunos ejemplos de este tipo de centros y de los materiales que utilizan pueden encontrarse en la University College London (http://writingcentre.ioe.ac.uk/), la Harvard University (http://writingcenter.fas.harvard. edu/) o las Universidades de Navarra (http://www.unav.edu/web/centro-deescritura/home) o Ramón Llull (http://recerca.blanquerna.edu/estrategiaslectura-escritura-academica/recursos/), entre otras.

De manera complementaria al trabajo institucional, el proceso de enculturización descansa en gran medida en la labor tutorial que realizan los profesores. La construcción del pensamiento disciplinar se requiere un diálogo entre el tutor-lector y el alumno-escritor. El producto de ese diálogo es un aprendizaje por parte del alumno de las convenciones académicas, adaptando las características retóricas de la escritura a su pregunta de investigación y su enfoque metodológico (Russell, 1997). 
La toma de conciencia de las distintas modalidades de TFG y TFM no está relacionada con las guías de escritura académica. En realidad, dicha conciencia de los prototipos de géneros discursivos académicos debe ser parte del currículum de las distintas disciplinas del Grado o del propio Máster. Para ello, Tardy (2009) propone crear entornos donde los alumnos tengan acceso a las estrategias y fuentes que les permitan acceder a diversos géneros académicos, desarrollar tareas que posibiliten el acceso a dichos géneros $\mathrm{y}$, por último, generar conocimiento disciplinar partiendo de las relaciones que dichos conocimientos tienen con determinados géneros discursivos académicos concretos.

En conclusión, la complejidad asociada a la construcción de la identidad académica requiere que el proceso de alfabetización académica sea iniciado desde el primer año del periodo de la formación universitaria. La formación y evaluación en las distintas asignaturas que componen un plan de estudios debería incorporar la escritura académica. En este sentido, la única manera de pertenecer a una comunidad de prácticas es la introducción a través de diversas y heterogéneas prácticas de socialización académica (Atkinson, 1999). 


\section{REFERENCIAS BIBLIOGRÁFICAS}

AA.VV. (2010). Monográfico La evaluación de competencias. REDU. Revista de Docencia Universitaria, 8(1). Recuperado de http://redu.net/redu/documentos/vol8_n1_completo.pdf

Álvarez, M. \& Yániz, C. (2015). Writing practices in Spanish university. Cultura y Educación, 27(3), 594-628.

ANECA (s.f.). Guía de apoyo para la redacción, puesta en práctica y evaluación de los resultados de aprendizaje. Recuperado de https://goo.gl/rFWdIR.

AQU (2013). Guía para la acreditación de las titulaciones oficiales de grado y máster. Recuperado de http://goo.gl/uz3AP0

Atkinson, D. (1999). Scientific discourse in sociohistorical context. The philosophical transactions of the Royal Society of London, 1675-1975. Mahwah, NJ: Lawrence Erlbaum.

Barton, D. (1991). The social nature of writing. En D. Barton \& R. Ivanič (Eds.), Writing in the community (pp. 1-13). London: Sage Publications.

Barton, D. (1994). Literacy: An introduction to the ecology of written language. Oxford: Blackwell.

Barton, D., Hamilton, M. \& Ivanič, R. (Eds.) (2000). Situated Literacies: Reading and Writing in Context. LondonNew York: Routledge.

Bazerman, Ch., Littele, J., Bethel, L., Chavkin, T., Fouquette, D., \& Garufis, J. (2005). Reference guide to writing across the curriculum. West Lafayette, Indiana: Parlos Press/The WAC Clearinghouse.

Carlino, P. (2013). Alfabetización académica diez años después. Revista Mexicana de Investigación Educativa, 18(57), 355-381.
Carlino, P., Iglesia, P., y Laxalt, I. (2013). Concepciones y prácticas declaradas de profesores terciarios en torno al leer y escribir en las asignaturas. REDU. Revista de Docencia Universitaria, 11(1), 105-136.

Carter, M., Ferzli, M., \& Weibe, E. (2004). Teaching genre to English firstlanguage adults: A study of the laboratory report. Research in the Teaching of English, 38(4), 395-413.

Corcelles, M., Oliva, A., Castelló, M., \& Milian, M. (2015). Writing at university: are we on the same page? Cultura y Educación, 27(3), 534-568.

Devitt, A. J. (2004). Writing genres. Carbondale: Southern Illinois University Press.

ECA (s.f.). Principles regarding outcomes in accreditation procedures. Recuperado de http://goo.gl/oPXwhC

English, F. (2011). Students writing and genre. Reconfiguring academic knowledge. London/New York: Continuum.

European Consortium for Accreditation in Higher Education (ECA) (2012). Principles regarding learning outcomes in acreditation procedures. Recuperado de http://ecahe.eu/w/index.php/Principles_regarding_learning_outcomes_ in_accreditation_procedures

Fundación Madrid (2014). Guía de evaluación para la renovación de la acreditación de títulos oficiales de Grado y Máster. Recuperado de http://goo.gl/ doJS2u.

Fairclough, N. (1989). Language and power. London/New York: Longman.

Gee, J. (1996). Social Linguistics and Literacies: Ideology and Discourses. 
Critical Perspectives on Literacy and Education. Bristol, PA: Falmer Press.

Ganobcsik-Williams, L. (Ed.) (2006). Teaching academic writing in UK Higher Education. Theories, practices and models. New York: Palgrave MacMillan.

Gardner, S. \& Nesi, H. (2013). A classification of genre families in university student writing. Applied Linguistics, 34, 25-52. Doi:10.1093/aplin/ams024

Guzmán-Simón, F. y García-Jiménez, E. (2014). Los hábitos lectoescritores en los alumnos universitarios. Revista Electrónica Interuniversitaria de Formación del Profesorado, 17(3), 79-92. Doi: http://dx.doi.org/10.6018/reifop.17.3.204071

Guzmán-Simón, F. \& García-Jiménez, E. (2015). The Academic Literacy at the University: A predictive Study. $R E$ LIEVE, 21(1), art. ME3. DOI: 10.7203/ relieve.21.1.5018

Hyland, K. (2009). Academic discourse. English in a global context. London/ New York: Continuum.

Ivanič, R. (1998). Writing and identity. The discoursal construction of identity in academic writing. Amsterdam: John Benjamins.

Ivanič, R., Edwards, R., Barton, D., Martin-Jones, M., Fowler, Z., Hughes, B., Mannion, G., Miller, K., Satchwell, C., \& Smith, J. (2009). Improving learning in College. Rethinking literacies across the curriculum. London/New York: Routledge.

Kress, G. (2003). Literacy in the New Media Age. London: Routledge.

Kress, G. \& Van Leeuwen, T. (1996). Reading Images: The Grammar of Visual Design. London: Routledge.
Kruse, O. (2013). Perspectives on academic writing in European Higher Education: Genres, practices and competences. REDU. Revista de Docencia Universitaria, 11(1), 37-58.

Kruse, O. \& Chitez, M. (2012). Contrastive genre mapping in academic contexts: An intercultural approach. Journal of Academic Writing, 2(1), 59-73.

Lea, M. R. \& Street, V. (1998). Student writing in higher education: An academic literacies approach. Studies in Higher Education, 23(2), 157-172.

Lea, M. \& Street, B. (2006). The «Academic literacies» Model: Theory and Applications. Theory into Practice, 45(4), 368-377.

Lillis, Th. (2001). Student writing. Access, regulation, desire. London/New York: Routledge.

Lillis, Th., Harrington, K., Lea, M. R., \& Mitchell, S. (Eds.) (2015). Working with academic literacies: Case studies towards transformative practice. Fort Collins, Colorado/Anderson, South Carolina: The WAC Clearinghouse/ Parlor Press.

Nesi, H. \& Gardner, Sh. (2012). Genre across the disciplines. Student writing in higher education. Cambridge: Cambridge University Press.

Prior, P. (1998). Writing/Disciplinarity. A sociohistoric account of literate activity in the academy. New York/London: Routledge.

Prior, P. \& Bilbro, R. (2012). Academic enculturation: Developing literate practices and disciplinary identities. En M. Castelló y Ch. Donahue (Eds.), University writing: Selves and texts in Academic Societies (pp. 20-31). Bingley: Emerald.

Real Decreto 1393/2007, de 29 de octubre, por el que se establece la ordenación de las enseñanzas universitarias 
oficiales. BOE 260 , de 30 de octubre de 2007, páginas 4403-44068.

Real Decreto 861/2010, de 2 de julio, por el que se modifica el Real Decreto 1393/2007, de 29 de octubre, por el que se establece la ordenación de las enseñanzas universitarias oficiales. BOE 161, de 3 de julio de 2010, páginas 58454-58468.

Real Decreto 1027/2011, de 15 de julio, por el que se establece el Marco Español de Cualificaciones para la Educación Superior. BOE 185, de 3 de agosto de 2011, páginas 87912-98918.

Rodríguez Gómez, G. e Ibarra Sáez, M. (Eds.) (2011). E-evaluación orientada al e-aprendizaje estratégico en la educación superior. Madrid: Narcea.

Russell, D. R. (1997). Rethinking genre in school and society: An activity theory analysis. Written Communication, 14, 504-554. DOI: 10.1177/0741088397014004004

Russell, D. R. \& Yañez, A. (2003). 'Big picture people rarely become historians': Genre systems and the contradictions of general education. En Bazerman, C. y Russell, D. R. (Eds.), Writing selves/Writing societies. Research from activity perspectives ( $\mathrm{pp}$. 331-362). Fort Collins/La Jolla: The WAC Clearinghouse.

Russell, D. R., Lea, M., Parker, J., Street, B., y Donahue, T. (2009). Exploring notions of genre in «Academic literacies» and "Writing across the curriculum»: Approaches across countries and contexts. En C. Bazerman, A. Bonini, y D. Figueiredo (Eds.), Genre in a changing world. Perspectives on writing (pp. 395-423). Colorado: Parlor Press/ WAC Clearinghouse.

Street, B. (1984). Literacy in theory and practice. Cambridge: Cambridge University Press.

Street, B. (2004). Academic literacies and the 'new orders': implications for research and the practice in student writing in Higher Education. Learning and Teaching in the Social Sciences, 1(1), 9-20. DOI: 10.1386/ltss.1.1.9/0

Swales, J. M. (1990). Genre analysis. English in academic and research settings. Cambridge: Cambridge University Press.

Swales, J. M. (2004). Research genres. Explorations and applications. Cambridge: Cambridge University Press.

Tardy, Ch. M. (2009). Building genre knowledge. West Lafayette, Indiana: Parlor Press.

Wingate, U. \& Tribble, C. (2012). The best of both worlds? Towards an English for Academic Purposes/ Academic Literacies writing pedagogy. Studies in Higher Education, 37(4), 481-495. 


\section{PERFIL ACADÉMICO Y PROFESIONAL DE LOS AUTORES}

Eduardo García-Jiménez. Catedrático de Métodos de Investigación y Diagnóstico en Educación en la Universidad de Sevilla y miembro del grupo de investigación EVALfor: Evaluación en contextos formativos (SEJ-509). En los últimos años sus trabajos se han centrado en el desarrollo de procedimientos e instrumentos de evaluación en educación, a través de aplicaciones informáticas, para uso del profesorado que trabaja en diferentes etapas educativas tales como EVALCOMIX, DIPEVAL y HEVAFOR.

Fernando Guzmán Simón. Profesor de Didáctica de la Lengua y la Literatura en la Facultad de Ciencias de la Educación de la Universidad de Sevilla. Su investigación se centra en la evaluación de las habilidades lingüísticas en Educación Infantil, del desarrollo de la multialfabetización y de los procesos de alfabetización académica. Es miembro del grupo de investigación EVALfor: Evaluación en contextos formativos (SEJ-509).

Dirección de los Autores: Eduardo García-Jiménez

Universidad de Sevilla Facultad de Cien-

cias de la Educación Departamento de

Métodos de Investigación y Diagnóstico en

Educación

E-mail: egarji@us.es

Fernando Guzmán-Simón

Universidad de Sevilla Facultad de Cien-

cias de la Educación Departamento de

Didáctica de la Lengua y la Literatura y

Filologías Integradas

E-mail: fernandoguzman@us.es

Fecha Recepción del Artículo: 9. Diciembre. 2015

Fecha Modificación del Artículo: 28. Enero. 2016

Fecha Aceptación del Artículo: 29. Enero. 2016

Fecha Revisión para publicación: 15. Febrero. 2016 
